\title{
Mass spectrometry beyond the native state
}

\author{
Shane A. Chandler \& Justin L.P. Benesch* \\ Department of Chemistry, Physical \& Theoretical Chemistry Laboratory, University of Oxford, \\ OXford, OX1 3QZ, U.K.
}

*Correspondence to justin.benesch@chem.ox.ac.uk, +44 1865285420 


\begin{abstract}
Native mass spectrometry allows the study of proteins by probing in vacuum the interactions they form in solution. It is a uniquely useful approach for structural biology and biophysics due to the high resolution of separation it affords, allowing the concomitant interrogation of multiple protein components with high mass accuracy. At its most basic, native mass spectrometry simply reports the mass of intact proteins and the assemblies they form in solution. However, the opportunities for more detailed characterisation are extensive, enabled by the exquisite control of ion motion that is possible in vacuum. Here we describe recent developments in mass spectrometry approaches to the structural interrogation of proteins both in, and beyond, their native state.
\end{abstract}

\title{
Highlights
}

- Native mass spectrometry allows the interrogation of protein conformational space

- Manipulation of charge populates different minima in the protein folding funnel

- Gas-phase activation allows trajectories out of the folding funnel to be investigated

- Labelling provides an additional dimension for probing protein surfaces in vacuum

- Spectroscopy and imaging promise direct high-resolution structural information 


\section{Main text}

\section{Introduction}

Native mass spectrometry (MS) is the study of proteins and other biomolecules in the vacuum of a mass spectrometer, having initially maintained their noncovalent interactions upon transferral from solution [1,2]. A variety of experimental data and simulations have shown that the native state of proteins and their complexes can persist on the millisecond timescale of MS experiments $[3,4]$. This provides ample opportunity for interrogation of not just native structure, but also the free-energy landscape accessible to the protein $[5,6]$. This can be viewed in terms of a canonical protein (un)folding funnel, where protein ions can populate states differing in their atomic coordinates and energy (Fig. 1). While the native states of proteins are relatively accessible to structural biologists and biophysicists, probing states populated only to low levels at equilibrium, or fleetingly during assembly and folding, remains extremely challenging [7]. Here we discuss how and why MS-based experiments can contribute to this endeavour.

We first consider how the protein folding funnel can be explored within the mass spectrometer, discussing the role played by protein charge state in populating different minima. We evidence how these represent starting points for exploring higher levels of the protein folding funnel by activating ions within the mass spectrometer. In the second part of this review, we describe emerging gas-phase labelling, spectroscopy, and imaging methods that are being integrated with native MS. These approaches promise to provide unprecedented detail regarding the structure of proteins in vacuum, including during excursions from the native state. 


\section{Coulombic exploration of the free energy landscape}

Protein ions generated by (nano)electrospray ionisation each populate a distribution of charge states. Oftentimes the gross structure, evidenced by collision cross-section (CCS) values obtained from ion mobility (IM) measurements, appears to be unaffected by this charge state variation [8]. However, in cases where the range of charge states populated is broad relative to the average, or when the protein has an intrinsic tendency towards disorder, significant variations in CCS are observed $[9,10]$. This demonstrates how, as in solution [11], different charge states of a protein in vacuum correspond to different freeenergy minima in the free energy landscape (Fig. 1A).

To investigate how global protein structure depends on charge state, a number of recent studies have performed IM-MS on protein ions that are selected within the mass spectrometer. Charge manipulation has been performed via ion-ion collisions with a reaction partner of opposite polarity, most commonly performed by abstraction of a proton $[12,13]$, or transfer of an electron $[14,15]$. A number of studies have shown that reduction of charge on native conformers of ubiquitin and cytochrome $\mathrm{C}$ results in a stepwise compaction [13-18]. In other cases, increases in CCS upon charge reduction are observed $[14,19]$. This demonstrates the impact of charge on the initial position on the folding funnel, and that other local minima can be occupied by charge modulation. These minima therefore represent alternative starting points for exploration of the conformational landscape.

In order to navigate the protein folding funnel, the ions can be activated within the mass spectrometer (Fig. 1), typically by energetic collisions with a bath gas. By varying the amount of energy supplied, the types of bonds broken and level of structural information obtained can be controlled. At a low level of gas-phase activation, proteins first undergo structural rearrangements and unfolding due to breakage of intramolecular non-covalent 
bonds [20]. IM-MS measurements have shown that this occurs via a number of discrete transitions in CCS (Fig. 2A), diagnostic of the trajectory taken by the protein as it escapes the folding funnel. The structural evolution of a protein depends strongly on its charge state $[15,17,21]$, and tandem IM-MS experiments reveal complex conformational interdependencies [22], consistent with the view of different minima in the folding funnel being populated and leading to alternative pathways out (Fig. 1).

As a consequence, the transitions observed and the energies at which they occur represent a characteristic signature for the protein charge state being investigated. This can be exploited as a fingerprint for comparison, enabling, for instance, distinction between different antibody structures and interactions [23-26]. Unfolding has also been actively pursued as a measure of ligand-stabilisation of protein structure [27], revealing measures consistent with solution data [28], as well as reflecting interactions that are strengthened in vacuum [29]. The number of unfolding transitions appears to reflect the number of independent structural domains seen in solution [30]. However, assignment of the transitions observed to individual regions of the protein is difficult. One way to address this is to use specific small-molecule probes, and correlate their release upon activation with the transitions in CCS (Fig. 2B)[31]. Potentially a more general strategy will come from performing electron-mediated fragmentation of the protein during its unfolding pathway [32].

Increasing activation further results in the dissociation of non-covalently attached units, protein subunits or other ligands. In general, dissociation of multimeric assemblies appears to proceed via the expulsion of single, highly charged subunits [20]. The identity of the dissociation products can yield compositional information on the complex, while the asymmetric partitioning of charge between products can be exploited to mine spectra not resolvable by MS alone [33-35]. However, fast activation, in particular by collision 
with a surface, can lead to information regarding the assembly and architecture of the complex [36,37]. This can be rationalised by the activation allowing access to the higher reaches of the protein energy landscape without transitioning through lower-energy intermediates via polypeptide unfolding [38]. Remarkably, this information appears to be retained on the timescale of seconds [39].

At the highest energies accessible in the mass spectrometer, covalent fragmentation of the polypeptide backbone can be induced (Fig. 2A)[20]. This means that "top-down" proteomics is possible from the starting point of a native protein assembly [40]. Exploiting this capability enables the attribution of properties of individual protein assemblies to differences in their primary sequence [41]. A particularly attractive means for reducing native proteins into peptide fragments comes from irradiation of a protein complex using UV photons [42]. This has been shown to operate on a fast timescale, providing comprehensive sequence coverage $[43,44]$, while retaining non-covalent interactions [43] and promoting a more symmetric dissociation pathway [45]. Interestingly, regions of enhanced fragmentation correlate reasonably well with solvent exposure in solution suggesting that aspects of the native fold are retained (Fig 2C)[46]. Similar correlations have also been found when using electron-mediated fragmentation $[32,47]$.

\section{Structural interrogation of native and activated states}

Aside from mass and charge, the information most frequently obtained from native MS experiments is CCS, through the implementation of an orthogonal IM dimension [48]. While the CCS contains significant information on the global shape of proteins $[49,50]$, and can be used to aid structural modelling [51,52], complementary approaches are 
needed to probe structure on a local level. An attractive strategy is to perform labelling experiments analogous to those in solution [53], in the vacuum of the mass spectrometer. This provides a means for probing the surface of a native protein, mass-separated from other species in solution. Furthermore, it can be used to interrogate the structures populated along the activation pathway, allowing for a detailed understanding of surface interactions and interfaces (Fig.2A, lower panels).

Gas-phase labelling strategies have mainly involved hydrogen-deuterium exchange (HDX) reactions by collisions between the protein ion and a deuterated vapour [54]. The level of deuterium uptake, which manifests itself as a mass increase, has been shown to be sensitive to protein conformation in solution [55-57], charge state [56,58], and structural changes due to collisional activation [59]. Differences in deuterium uptake between a complex and its constituents are a measure of the occlusions and interactions at the interface. By dissociating the labelled complex to examine the partitioning of uptake over the constituents, information as to the associations made can be obtained (Fig. 2D)[60]. This experiment relies on the activation not causing significant "scrambling" of deuterium labels between the dissociation products, a phenomenon that poses a challenge for increasing the spatial resolution of solution-phase HDX [61]. However, it appears that scrambling across non-covalent interfaces is relatively minimal [62].

To extract the maximal amount of information from a labelling experiment requires localisation of the labels to individual amino acids. This is in principle possible using a top-down strategy, with the proviso that the activation that produces the peptide fragments outpaces any proton scrambling. The prospect of achieving this comes from the coupling of fast activation techniques with gas-phase HDX [63-66], but has yet to be explored fully on native proteins and assemblies. 
An alternative means to probing protein structure in vacuum directly is afforded by spectroscopic studies. Notably, gas-phase IR spectra display distinguishing features that mirror those obtained from measurements in solution. This has been exploited to reveal the retention of protein secondary structure in native charge states [67], and the formation of new structures upon activation [68], while measurements on green fluorescent protein allowed the role of solvent on the chromophore to be probed [69]. Spectroscopic methods also have the potential for reporting intra-molecular distances. For instance, recent data has shown that Förster resonance energy transfer experiments in the gas phase are feasible [70,71]. A related means to obtain distance measures, which has the benefit of not requiring the addition of an extrinsic fluorescent label, comes from taking advantage of excitation energy transfer [72]. By studying disulphide bond cleavage, the proximity of aromatic amino acids can be determined through residuespecific absorption behaviour [73].

Though many of the techniques discussed above push MS-based methodologies towards revealing more structural information, the ideal would be to directly image individual proteins in the gas phase, rather than inferring structure from an ensemble. The attraction from an imaging perspective is the isolation of the protein, and the control afforded in the gas phase. Recent studies show that this ideal is not unrealistic (Fig. 3). Intact protein and protein complex ions can be mass selected for "soft landing" onto a surface for imaging [74]. While imaging the native structure is of obvious interest, the technique can also report on the conformational space accessible to the proteins as a function of charge state (Fig. 3A)[75]. Landing native ions can also preserve intermolecular non-covalent interactions, with electron microscopy revealing structural differences between mass-selected species (Fig. 3B)[76]. Recently, low-energy electron holography has achieved sub-nanometre resolution on single molecules, soft-landed onto 
ultra-clean graphene surfaces. Strikingly, native orientations of the landed proteins can be clearly seen (Fig. 3C)[77]. An alternative approach, that does not require deposition on a surface, is to use an X-ray free electron laser which allows for the diffraction data from individual molecules to be obtained before their destruction. The current state-of-the-art is $\approx 20$-nm resolution, but nonetheless different orientations of reconstructed single particle images of carboxysomes show good agreement with predicted geometric models (Fig. 3D)[78]. These studies highlight how the integration of imaging approaches with the selection capabilities, and potentially ion alignment [79], of MS could enable novel methods for high-resolution structure determination.

\section{Perspective}

In the 25 years since its inception, native MS has become an important approach for structural biology, and enabled many investigations into the structure that proteins populate at equilibrium. Here we have presented the case that this approach has great potential, some of which has already been realised, for probing the conformational space of proteins. However, while the preservation of native structure in the gas phase is clear, it remains to be seen to what extent the free-energy landscape resembles that in solution. In the absence of water, and consequently any competition between protein-solvent and intra-molecular solvation, the landscape will be more rugged, with higher barriers to conformational inter-conversion [5]. However, an important question is to what extent gas-phase trajectories in the protein folding funnel resemble the functional excursions from the native state in solution. Interestingly, it has been shown that, in protein crystals, mechanics stimulated by the application of external electric fields that act on local dipoles resemble the biologically relevant motions [80]. This suggests that exploration of the protein folding funnel in vacuum, where Coulombic interactions are dominant, will likely 
encompass functional trajectories. The literature we have highlighted here reveals a key aspect of native MS, namely that the control afforded by ion manipulation in vacuum allows the implementation of multiple experiments on the same ion. The versatility, and multi-pronged nature of the approach will be key in probing the free-energy landscape of proteins.

\section{Acknowledgments}

We thank Jennifer Brodbelt, Brandon Ruotolo, Kasper Rand, Stephan Rauschenbach and Max Hantke for providing and discussing material for Figures 2 and 3. SAC is funded by an iCASE studentship from the Biotechnology and Biological Sciences Research Council and Waters Corp (BB/L017067/1). 


\section{References}

1. Leney AC, Heck AJR: Native Mass Spectrometry: What is in the Name? Journal of The American Society for Mass Spectrometry 2017, 28:5-13.

2. Winston RL, Fitzgerald MC: Mass spectrometry as a readout of protein structure and function. Mass Spectrom Rev 1997, 16:165-179.

3. Hilton GR, Benesch JLP: Two decades of studying non-covalent biomolecular assemblies by means of electrospray ionization mass spectrometry. $J$ R Soc Interface 2012, 9:801-816.

4. Meyer T, Gabelica V, Grubmller H, Orozco M: Proteins in the gas phase. Wiley Interdisciplinary Reviews: Computational Molecular Science 2013, 3:408--425.

5. Clemmer DE, Russell DH, Williams ER: Characterizing the Conformationome: Toward a Structural Understanding of the Proteome. Accounts of chemical research 2017, 50:556--560.

6. Wyttenbach T, Pierson NA, Clemmer DE, Bowers MT: Ion mobility analysis of molecular dynamics. Annu Rev Phys Chem 2014, 65:175-196.

7. Dill KA, MacCallum JL: The protein-folding problem, 50 years on. Science 2012, 338:1042-1046.

8. Allen SJ, Schwartz AM, Bush MF: Effects of polarity on the structures and charge states of native-like proteins and protein complexes in the gas phase. Anal Chem 2013, 85:12055-12061.

9. Borysik AJ, Kovacs D, Guharoy M, Tompa P: Ensemble Methods Enable a New Definition for the Solution to Gas-Phase Transfer of Intrinsically Disordered Proteins. J Am Chem Soc 2015, 137:13807-13817.

10. Jhingree JR, Bellina B, Pacholarz KJ, Barran PE: Charge Mediated Compaction and Rearrangement of Gas-Phase Proteins: A Case Study Considering Two 
Proteins at Opposing Ends of the Structure-Disorder Continuum. J Am Soc Mass Spectrom 2017, 28:1450-1461.

11. Gitlin I, Carbeck JD, Whitesides GM: Why are proteins charged? Networks of charge-charge interactions in proteins measured by charge ladders and capillary electrophoresis. Angewandte Chemie-International Edition 2006, 45:3022-3060.

12. Laszlo KJ, Bush MF: Analysis of Native-Like Proteins and Protein Complexes Using Cation to Anion Proton Transfer Reactions (CAPTR). Journal of the American Society for Mass Spectrometry 2015, 26:2152-2161.

13. Zhao Q, Soyk MW, Schieffer GM, Fuhrer K, Gonin MM, Houk RS, Badman ER: An Ion Trap-Ion Mobility-Time of Flight Mass Spectrometer with Three Ion Sources for Ion/Ion Reactions. Journal of the American Society for Mass Spectrometry 2009, 20:1549-1561.

14. Jhingree JR, Beveridge R, Dickinson ER, Williams JP, Brown JM, Bellina B, Barran PE: Electron transfer with no dissociation ion mobility-mass spectrometry (ETnoD IM-MS). The effect of charge reduction on protein conformation. International Journal of Mass Spectrometry 2017, 413:43-51.

15. Lermyte F, Lacki MK, Valkenborg D, Gambin A, Sobott F: Conformational Space and Stability of ETD Charge Reduction Products of Ubiquitin. J Am Soc Mass Spectrom 2017, 28:69-76.

16. Laszlo KJ, Buckner JH, Munger EB, Bush MF: Native-Like and Denatured Cytochrome c Ions Yield Cation-to-Anion Proton Transfer Reaction Products with Similar Collision Cross-Sections. Journal of The American Society for Mass Spectrometry 2017, 28:1382-1391. 
17. Laszlo KJ, Munger EB, Bush MF: Folding of Protein Ions in the Gas Phase after Cation-to-Anion Proton-Transfer Reactions. J Am Chem Soc 2016, 138:95819588.

18. Zhao Q, Schieffer GM, Soyk MW, Anderson TJ, Houk RS, Badman ER: Effects of ion/ion proton transfer reactions on conformation of gas-phase cytochrome c ions. J Am Soc Mass Spectrom 2010, 21:1208-1217.

19. Laszlo KJ, Bush MF: Interpreting the Collision Cross Sections of Native-like Protein Ions: Insights from Cation-to-Anion Proton-Transfer Reactions. Analytical Chemistry 2017, 89:7607-7614.

20. Benesch JLP: Collisional activation of protein complexes: picking up the pieces. Journal of the American Society for Mass Spectrometry 2009, 20:341-348.

21. Wagner N, Clemmer DE, Russell DH: ESI-IM-MS and Collision-Induced Unfolding Provide Insight into the Linkage Dependent Interfacial Interactions of Covalently Linked Diubiquitin. Analytical Chemistry 2017:acs.analchem.7b02932.

22. Koeniger SL, Clemmer DE: Resolution and structural transitions of elongated states of ubiquitin. Journal of the American Society for Mass Spectrometry 2007, 18:322-331.

23. Botzanowski T, Erb S, Hernandez-Alba O, Ehkirch A, Colas O, Wagner-Rousset E, Rabuka D, Beck A, Drake PM, Cianferani S: Insights from native mass spectrometry approaches for top- and middle- level characterization of sitespecific antibody-drug conjugates. MAbs 2017, 9:801-811.

24. Huang Y, Salinas ND, Chen E, Tolia NH, Gross ML: Native Mass Spectrometry, Ion mobility, and Collision-Induced Unfolding Categorize Malaria Antigen/Antibody Binding. J Am Soc Mass Spectrom 2017. 
25. Pacholarz KJ, Peters SJ, Garlish RA, Henry AJ, Taylor RJ, Humphreys DP, Barran PE: Molecular Insights into the Thermal Stability of mAbs with VariableTemperature Ion-Mobility Mass Spectrometry. Chembiochem : a European journal of chemical biology 2016, 17:46-51.

26. Tian Y, Han L, Buckner AC, Ruotolo BT: Collision Induced Unfolding of Intact Antibodies: Rapid Characterization of Disulfide Bonding Patterns, Glycosylation, and Structures. Analytical Chemistry 2015, 87:11509-11515.

27. Allison TM, Reading E, Liko I, Baldwin AJ, Laganowsky A, Robinson CV: Quantifying the stabilizing effects of protein-ligand interactions in the gas phase. Nature Communications 2015, 6:8551.

28. Laganowsky A, Reading E, Allison TM, Ulmschneider MB, Degiacomi MT, Baldwin AJ, Robinson CV: Membrane proteins bind lipids selectively to modulate their structure and function. Nature 2014, 510:172-175.

29. Landreh M, Marklund EG, Uzdavinys P, Degiacomi MT, Coincon M, Gault J, Gupta K, Liko I, Benesch JLP, Drew D, et al.: Integrating mass spectrometry with MD simulations reveals the role of lipids in $\mathrm{Na}+\mathrm{H}+$ antiporters. Nat Commun 2017, 8:13993.

30. Zhong Y, Han L, Ruotolo BT: Collisional and Coulombic unfolding of gas-phase proteins: high correlation to their domain structures in solution. Angewandte Chemie (International ed. in English) 2014, 53:9209-9212.

31. Eschweiler JD, Martini RM, Ruotolo BT: Chemical probes and engineered constructs reveal a detailed unfolding mechanism for a solvent-free multidomain protein. Journal of the American Chemical Society 2017, 139:534540. 
32. Lermyte F, Sobott F: Electron transfer dissociation provides higher-order structural information of native and partially unfolded protein complexes. Proteomics 2015, 15:2813-2822.

33. Shepherd DA, Marty MT, Giles K, Baldwin AJ, Benesch JLP: Combining tandem mass spectrometry with ion mobility separation to determine the architecture of polydisperse proteins. International Journal of Mass Spectrometry 2014.

34. Wang G, de Jong RN, van den Bremer ETJ, Parren P, Heck AJR: Enhancing Accuracy in Molecular Weight Determination of Highly Heterogeneously Glycosylated Proteins by Native Tandem Mass Spectrometry. Anal Chem 2017, 89:47934797.

35. Marty MT, Hoi KK, Gault J, Robinson CV: Probing the Lipid Annular Belt by GasPhase Dissociation of Membrane Proteins in Nanodiscs. Angew Chem Int Ed Engl 2016, 55:550-554.

36. Quintyn RS, Yan J, Wysocki VH: Surface-induced dissociation of homotetramers with D2 symmetry yields their assembly pathways and characterizes the effect of ligand binding. Chemistry and Biology 2015, 22:583-592.

37. Song Y, Nelp MT, Bandarian V, Wysocki VH: Refining the Structural Model of a Heterohexameric Protein Complex: Surface Induced Dissociation and Ion Mobility Provide Key Connectivity and Topology Information. ACS Cent Sci 2015, 1:477-487.

38. Zhou M, Wysocki VH: Surface induced dissociation: dissecting noncovalent protein complexes in the gas phase. Acc Chem Res 2014, 47:1010-1018.

39. Harvey SR, Yan J, Brown JM, Hoyes E, Wysocki VH: Extended Gas-Phase Trapping Followed by Surface-Induced Dissociation of Noncovalent Protein Complexes. Anal Chem 2016, 88:1218-1221. 
40. Belov ME, Damoc E, Denisov E, Compton PD, Horning S, Makarov AA, Kelleher NL: From protein complexes to subunit backbone fragments: A multi-stage approach to native mass spectrometry. Analytical Chemistry 2013, 85:1116311173.

41. Ben-Nissan G, Belov ME, Morgenstern D, Levin Y, Dym O, Arkind G, Lipson C, Makarov AA, Sharon M: Triple-Stage Mass Spectrometry Unravels the Heterogeneity of an Endogenous Protein Complex. Analytical Chemistry 2017, 89:4708-4715.

42. Julian R: The Mechanism Behind Top-Down UVPD Experiments: Making Sense of Apparent Contradictions. Journal of The American Society for Mass Spectrometry 2017:1-4.

43. O'Brien JP, Li W, Zhang Y, Brodbelt JS: Characterization of native protein complexes using ultraviolet photodissociation mass spectrometry. Journal of the American Chemical Society 2014, 136:12920-12928.

44. Theisen A, Yan B, Brown JM, Morris M, Bellina B, Barran PE: Use of Ultraviolet Photodissociation Coupled with Ion Mobility Mass Spectrometry to Determine Structure and Sequence from Drift Time Selected Peptides and Proteins. Analytical Chemistry 2016, 88:9964-9971.

45. Tamara S, Dyachenko A, Fort KL, Makarov AA, Scheltema RA, Heck AJR: Symmetry of Charge Partitioning in Collisional and UV Photon-Induced Dissociation of Protein Assemblies. Journal of the American Chemical Society 2016, 138:1086010868.

46. Morrison LJ, Brodbelt JS: $193 \mathrm{~nm}$ Ultraviolet Photodissociation Mass Spectrometry of Tetrameric Protein Complexes Provides Insight into Quaternary and Secondary Protein Topology. Journal of the American Chemical Society 2016, 138:10849-10859. 
47. Li H, Wolff JJ, Van Orden SL, Loo JA: Native top-down electrospray ionization-mass spectrometry of $158 \mathrm{kDa}$ protein complex by high-resolution fourier transform ion cyclotron resonance mass spectrometry. Analytical Chemistry 2014, 86:317-320.

48. Lanucara F, Holman SW, Gray CJ, Eyers CE: The power of ion mobility-mass spectrometry for structural characterization and the study of conformational dynamics. Nat Chem 2014, 6:281-294.

49. Ewing SA, Donor MT, Wilson JW, Prell JS: Collidoscope: An Improved Tool for Computing Collisional Cross-Sections with the Trajectory Method. J Am Soc Mass Spectrom 2017, 28:587-596.

50. Marklund EG, Degiacomi MT, Robinson CV, Baldwin AJ, Benesch JLP: Collision cross sections for structural proteomics. Structure 2015, 23:791-799.

51. Ahdash Z, Pyle E, Politis A: Hybrid Mass Spectrometry: Towards Characterization of Protein Conformational States. Trends Biochem Sci 2016, 41:650-653.

52. Thalassinos K, Pandurangan AP, Xu M, Alber F, Topf M: Conformational States of macromolecular assemblies explored by integrative structure calculation. Structure 2013, 21:1500-1508.

53. Konermann L, Vahidi S, Sowole MA: Mass spectrometry methods for studying structure and dynamics of biological macromolecules. Anal Chem 2014, 86:213-232.

54. Maurer MM, Donohoe GC, Valentine SJ: Advances in ion mobility-mass spectrometry instrumentation and techniques for characterizing structural heterogeneity. Analyst 2015, 140:6782-6798. 
55. Beeston HS, Ault JR, Pringle SD, Brown JM, Ashcroft AE: Changes in protein structure monitored by use of gas-phase hydrogen/deuterium exchange. Proteomics 2015:2842-2850.

56. Rand KD, Pringle SD, Murphy JP, Fadgen KE, Brown J, Engen JR: Gas-phase hydrogen/deuterium exchange in a traveling wave ion guide for the examination of protein conformations. Anal Chem 2009, 81:10019-10028.

57. Zhu S, Campbell JL, Chernushevich I, Le Blanc JC, Wilson DJ: Differential Mobility Spectrometry-Hydrogen Deuterium Exchange (DMS-HDX) as a Probe of Protein Conformation in Solution. J Am Soc Mass Spectrom 2016, 27:991-999.

58. Donohoe GC, Khakinejad M, Valentine SJ: Ion mobility spectrometry-hydrogen deuterium exchange mass spectrometry of anions: part 1. Peptides to proteins. J Am Soc Mass Spectrom 2015, 26:564-576.

59. Valentine SJ, Clemmer DE: H/D Exchange Levels of Shape-Resolved Cytochrome c Conformers in the Gas Phase. Journal of the American Chemical Society 1997, 119:3558-3566.

60. Mistarz UH, Brown JM, Haselmann KF, Rand KD: Probing the Binding Interfaces of Protein Complexes Using Gas-Phase H/D Exchange Mass Spectrometry. Structure 2016, 24:310-318.

61. Rand KD, Zehl M, Jorgensen TJD: Measuring the Hydrogen/Deuterium Exchange of Proteins at High Spatial Resolution by Mass Spectrometry: Overcoming GasPhase Hydrogen/Deuterium Scrambling. Accounts of Chemical Research 2014, 47:3018-3027.

62. Modzel M, Stefanowicz P, Szewczuk Z: Hydrogen scrambling in non-covalent complexes of peptides. Rapid Commun Mass Spectrom 2012, 26:2739-2744. 
63. Khakinejad M, Kondalaji SG, Tafreshian A, Valentine SJ: Gas-Phase HydrogenDeuterium Exchange Labeling of Select Peptide Ion Conformer Types: a PerResidue Kinetics Analysis. Journal of The American Society for Mass Spectrometry 2015:1115-1127.

64. Mistarz UH, Brown JM, Haselmann KF, Rand KD: A Simple Setup for Gas-Phase H/D Exchange Mass Spectrometry Coupled to Electron Transfer Dissociation and Ion Mobility for Analysis of Polypeptide Structure on a Liquid Chromatographic Timescale. Analytical chemistry 2014, 86:11868-11876.

65. Pan JX, Heath BL, Jockusch RA, Konermann L: Structural Interrogation of Electrosprayed Peptide Ions by Gas-Phase H/D Exchange and Electron Capture Dissociation Mass Spectrometry. Analytical Chemistry 2012, 84:373378.

66. Rand KD, Pringle SD, Morris M, Brown JM: Site-Specific Analysis of Gas-Phase Hydrogen/Deuterium Exchange of Peptides and Proteins by Electron Transfer Dissociation. Analytical Chemistry 2012, 84:1931-1940.

67. Seo J, Hoffmann W, Warnke S, Bowers MT, Pagel K, von Helden G: Retention of Native Protein Structures in the Absence of Solvent: A Coupled Ion Mobility and Spectroscopic Study. Angewandte Chemie - International Edition 2016, 55:14173-14176.

68. Gonzalez Florez AI, Mucha E, Ahn DS, Gewinner S, Schollkopf W, Pagel K, von Helden G: Charge-Induced Unzipping of Isolated Proteins to a Defined Secondary Structure. Angew Chem Int Ed Engl 2016, 55:3295-3299.

69. Frankevich V, Barylyuk K, Chingin K, Nieckarz R, Zenobi R: Native biomolecules in the gas phase? The case of green fluorescent protein. Chemphyschem 2013, 14:929-935. 
70. Czar MF, Zosel F, Konig I, Nettels D, Wunderlich B, Schuler B, Zarrine-Afsar A, Jockusch RA: Gas-Phase FRET Efficiency Measurements To Probe the Conformation of Mass-Selected Proteins. Anal Chem 2015, 87:7559-7565.

71. Daly S, Poussigue F, Simon AL, MacAleese L, Bertorelle F, Chirot F, Antoine R, Dugourd P: Action-FRET: probing the molecular conformation of mass-selected gasphase peptides with Forster resonance energy transfer detected by acceptor-specific fragmentation. Anal Chem 2014, 86:8798-8804.

72. Hendricks NG, Lareau NM, Stow SM, McLean JA, Julian RR: Bond-specific dissociation following excitation energy transfer for distance constraint determination in the gas phase. Journal of the American Chemical Society 2014, 136:13363-13370.

73. Hendricks NG, Julian RR: Two-step energy transfer enables use of phenylalanine in action-EET for distance constraint determination in gaseous biomolecules. Chem. Commun. 2015, 51:12720-12723.

74. Rauschenbach S, Ternes M, Harnau L, Kern K: Mass Spectrometry as a Preparative Tool for the Surface Science of Large Molecules. Annual Review of Analytical Chemistry 2016, 9:473-498.

75. Rinke G, Rauschenbach S, Harnau L, Albarghash A, Pauly M, Kern K: Active Conformation Control of Unfolded Proteins by Hyperthermal Collision with a Metal Surface. Nano Letters 2014, 14:5609-5615.

76. Mikhailov VA, Mize TH, Benesch JLP, Robinson CV: Mass-selective soft-landing of protein assemblies with controlled landing energies. Analytical Chemistry 2014, 86:8321-8328. 
77. Longchamp J-N, Rauschenbach S, Abb S, Escher C, Latychevskaia T, Kern K, Fink H-W: Imaging proteins at the single-molecule level. Proceedings of the National Academy of Sciences 2017, 114:1474-1479.

78. Hantke MF, Hasse D, Maia FRNC, Ekeberg T, John K, Svenda M, Loh ND, Martin AV, Timneanu N, Larsson DSD, et al.: High-throughput imaging of heterogeneous cell organelles with an X-ray laser. Nature Photonics 2014, 8:943-949.

79. Marklund EG, Ekeberg T, Moog M, Benesch JLP, Caleman C: Controlling Protein Orientation in Vacuum Using Electric Fields. J Phys Chem Lett 2017, 8:45404544.

80. Hekstra DR, White KI, Socolich MA, Henning RW, Šrajer V, Ranganathan R: Electricfield-stimulated protein mechanics. Nature 2016, 540:400-405. 


\section{Highlighted references}

[31] Elegant study showing the elucidation of the gas-phase unfolding pathway for a multi-domained protein, by combining ion mobility and tandem mass spectrometry measurements on chemical-probe ligands.

[46] Demonstration of the versatility and applicability of UV photo-dissociation for probing native proteins via top-down mass spectrometry. The sensitivity of the approach to both tertiary and quaternary structure is evidenced.

[60] First demonstration of the use of gas-phase hydeogen-deuterium exchange to directly report on the binding interface in a protein-ligand complex. The authors demonstrate how uptake differences can report on the global structure, and localise contributions to the individual components in a way that reflects the interactions in the crystal structure.

[67] Report describing how the secondary structure of proteins can be obtained in the gas phase by IR action spectroscopy. As in solution, spectral features differ between the $\beta$-sheet protein $\beta$-lactoglobulin and $\alpha$-helical myoglobin, indicating that their conformations are retained in vacuum.

[73] Characterisation of the excitation energy transfer process for a peptide possessing Phe and Tyr residues. Understanding the energy transfer process, and its stong distance dependence, provides a valuable constraint for guiding molecular dynamics simulations.

[77] Demonstration of the use of low-energy electron holography at good resolution, on soft-landed 'native' proteins. A powerful demonstration on how three-dimensional imaging on a truly single molecule level can be realised. 
[17] Comparison of collision cross-section distributions for the products of proton transfer reactions on ubiquitin generated from denaturing conditions compared to the native structure. This work demonstrates the significant impact charge can play on ion structure and the conformational space an ion traverses.

[29] Combined experimental and simulation study revealing the structural rearrangements made by membrane proteins upon activation in the gas phase, and the origins of lipid-induced stabilisation.

[63] The gas-phase deuterium uptake of mobility-selected peptides is localised at a residue level using electron-mediated fragmentation. Demonstration of the detailed kinetic data that can be obtained by coupling rapid fragmentation to gas phase labelling.

[55] Gas-phase hydrogen-deuterium exchange is used to monitor protein structural pertubation in solution, and is shown to be sensitive to changes in secondary structure consistent with solution measurements.

[44] Selective dissociation of compact or extended conformations using UV photodissociation results in differing fragmentation efficiencies. Coupling ion mobility measurements provides further evidence for this method's sensitivity to secondary structure. 


\section{Figure legends}

\section{Figure 1}

Proteins exist on a rugged free-energy landscape in vacuum [5], that can be framed in terms of a protein folding funnel with the native state at the global minimum [7]. Different charge states of the protein can be considered as other minima on this surface, and thereby represent alternative starting points for exploration of the funnel. This can be achieved by gas-phase activation of the protein ions [20], enabling access to different unfolded states. At high levels of activation, dissociation of non-covalently bound units and even fragmentation of the polypeptide backbone can occur, providing information as to protein composition and identity.

\section{Figure 2}

Gas-phase activation and labelling are two orthogonal and complementary dimensions for interrogating the conformational states of proteins and trajectories in their folding funnel (Fig. 1).

A Activation provides structural information via the breaking of intra- and intermolecular bonds. This leads unfolding, dissociation of non-covalently bound ligands or subunits, and fragmentation of covalent bonds (upper row, left to right, and Fig. 1). These processes can provide information on the identity, composition and structure of native and non-native states. Labelling instead relies on the formation of new bonds at surfaceexposed sites. MS is sensitive to these reactions due to a shift in mass from the unlabelled species (dotted lines) upon introduction of the labelling agent. Labelling efficiency is sensitive to the nature of the species present and so differences can provide another means of informing on structure, across the activation spectrum (upper row, left to right). 
Some of the insights that can be obtained from gas phase activation, in the absence of presence of labelling agents, are inset, together with idealised schematics of anticipated IM-MS data.

B Gas-phase unfolding pathway of the three-domain human serum albumin upon collisional activation [30]. The degree of ligand dissociation over the unfolding trajectory was determined for five ligands known to bind individual domains specifically to assign specific regions of the protein to the transitions observed. The points at which $50 \%$ of each ligand (red, domain 1; green and yellow, domain 2; blue and cyan, domain 3) is removed are overlaid on the evolution of CCS with activation. Both ligands associated with domain 2 are lost prior to the formation of the first intermediate, suggesting that the first transition is coupled to this domain. The onset of domain 3 dissociation follows next, but the persistence of one of the ligands suggests only partial unfolding has occurred during the second transition. The third and final transition is attributed to both domains 2 and 3.

C UV photo-dissociation of streptavidin leads to both dissociation into subunits, and fragmentation of the backbone [46]. Dissociation is both symmetric (tetramer into dimers) and asymmetric (into monomer plus trimer). Mapping the fragmentation yield (blue) and the solvent-accessibility (red) as a function of residue number shows significant regions of overlap (purple). This provides evidence that fragmentation of the polypeptide backbone can inform on the level of protein tertiary structure.

D Gas-phase hydrogen-deuterium exchange of the trypsin-vasopressin complex allows the interrogation of the binding interface [60]. Greater deuterium uptake is observed when the components are labelled separately (78 and $5.5 \mathrm{Da}$, respectively) relative to the 
intact complex (76 Da), consistent with some exchangeable residues residing in the interface. Dissociation of the complex (spectrum shown), allowed for the individual uptake of each component to be determined, after having been labelled as part of an intact complex. The uptake differences can help inform on those residues involved in making inter-molecular contacts. (CID - collision induced dissociation).

\section{Figure 3}

Imaging methods can be coupled to native MS to provide high-resolution views of proteins separated in vacuum.

A Scanning tunnelling microscopy (STM) images of deposited cytochrome C ions shows a difference in unfolding as a function of charge state, with the highest charge states being almost completely extended [75].

B Transmission electron microscopy (TEM) images of mass-selected and soft-landed apoand holo-ferritin [76]. The crystal structure of the spherical ferritin cage is shown for comparison, and the cavity occupied with the iron core is illustrated.

C Low-energy electron holograms of bovine serum albumin after soft-landing, reveals images of individual molecules that correspond to the native structures [77].

D Reconstruction projection images of carboxysomes generated from single-particle diffraction patterns from an X-ray free electron laser (XFEL). Corresponding icosahedra are shown alongside in projection-matching orientations [78]. 
Figure 1

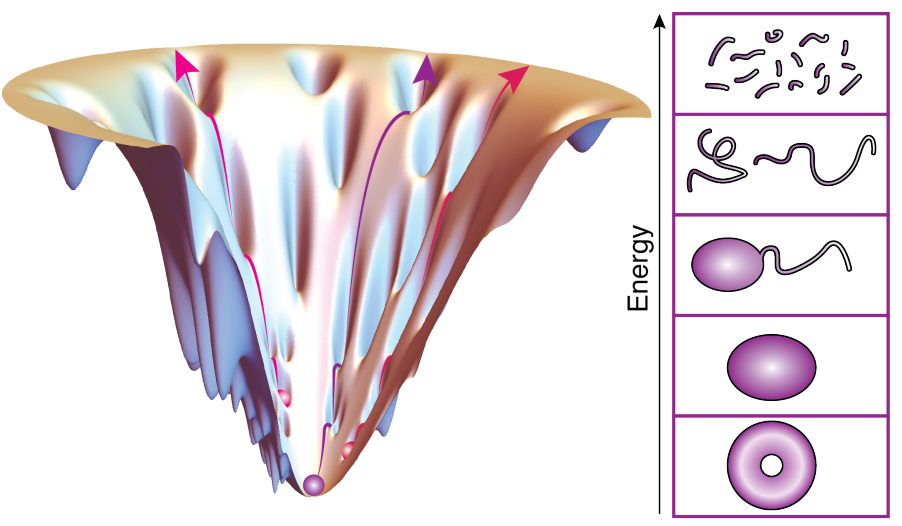


Figure 2

A

Gas-phase activation
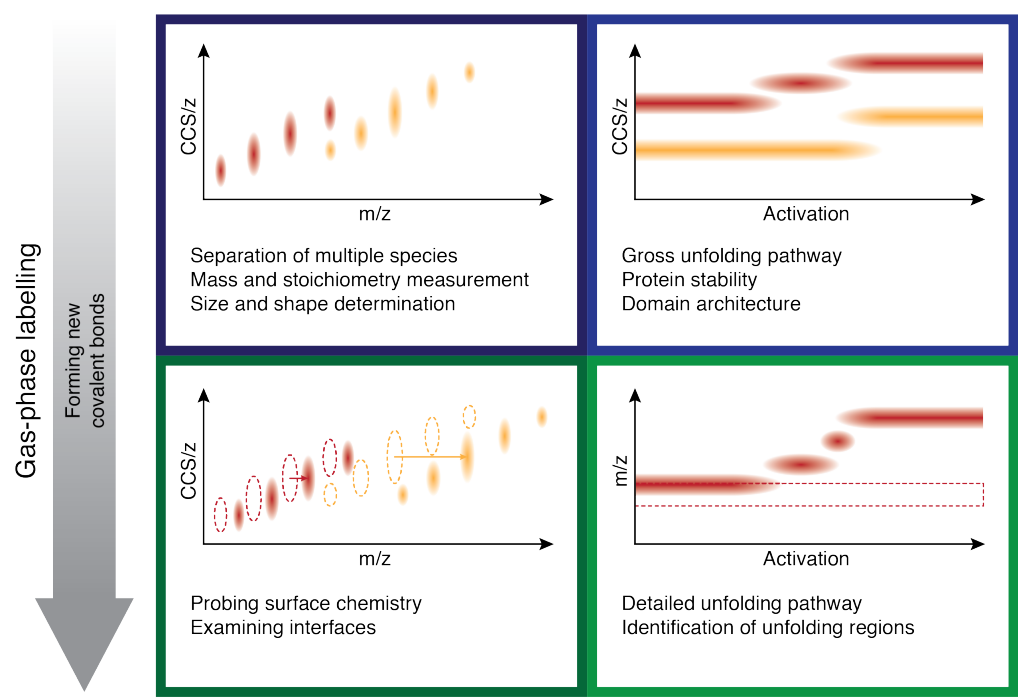

Maparation of multiple species

Mass and stoichiometry measuremen

Size and shape determination

Gross unfolding pathway

Protein stability

Domain architecture

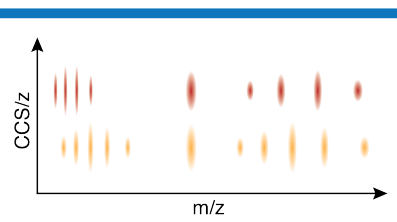

Identification of components

Deconvolution via charge reduction

Stability of interfaces

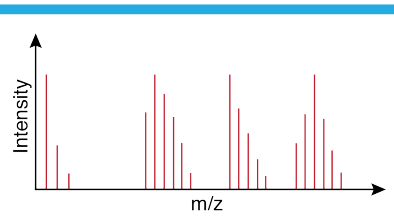

Protein identification

Mapping modifications

Mapping flexible regions

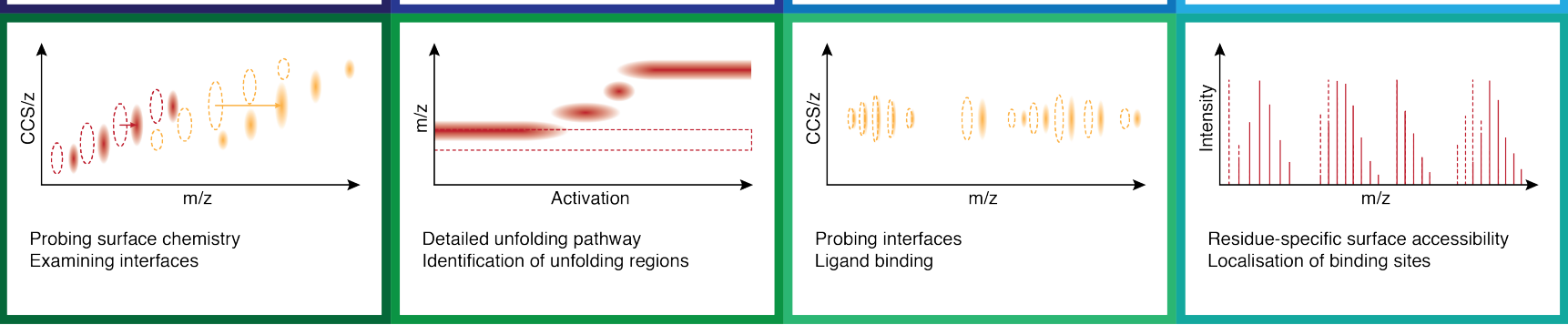

B

Native protein assembly

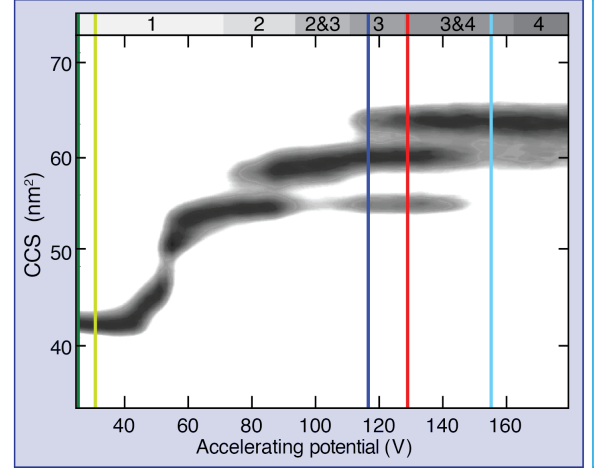

C

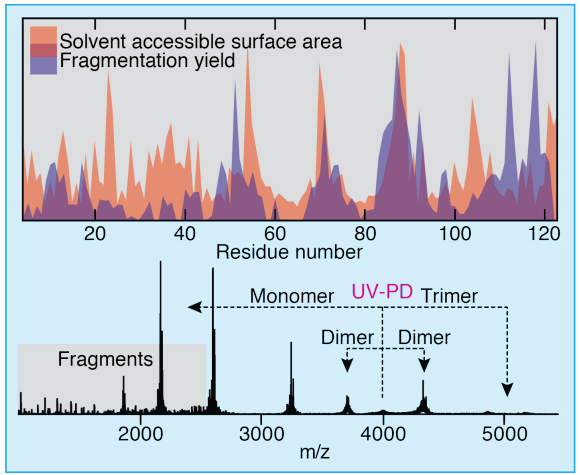

D

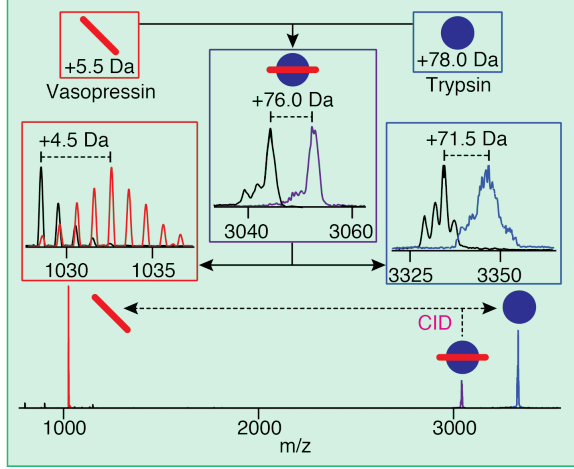


Figure 3

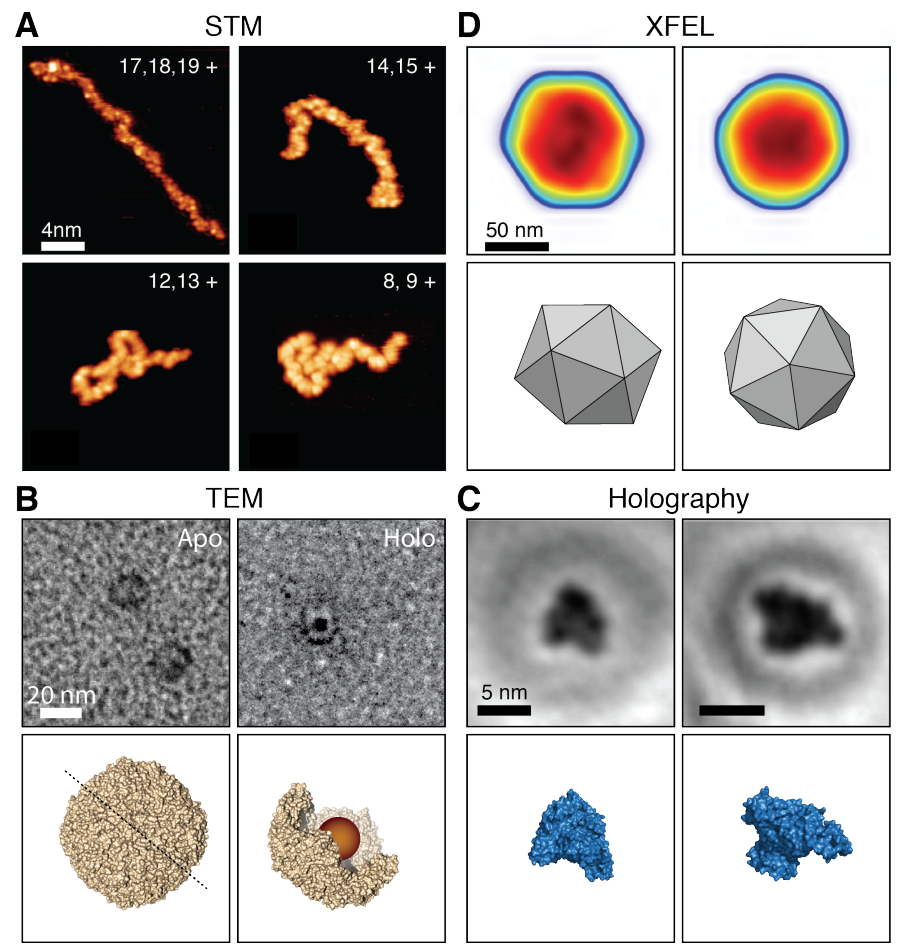

